\title{
The emerging role of interleukin-21 in allergic diseases (Review)
}

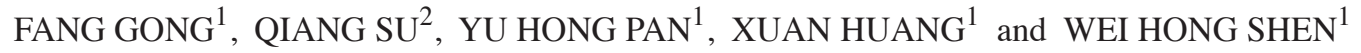 \\ Departments of ${ }^{1}$ Laboratory Medicine and ${ }^{2}$ Pulmonary Medicine, Allergy and Clinical Immunology, \\ The Third Hospital Affiliated to Nantong University, Wuxi, Jiangsu 214041, P.R. China
}

Received August 01, 2013; Accepted August 30, 2013

DOI: $10.3892 /$ br.2013.166

\begin{abstract}
Interleukin (IL)-21 is a newly discovered member of the type I cytokine family. Since its discovery in 2000, IL-21 has been the focus of extensive investigation, due to its homology to IL-2, IL-4 and IL-15 and its pleiotropic effects on innate and adaptive immune responses. An increasing amount of experimental evidence supports a role for IL-21 in the pathogenesis of several allergic diseases, such as allergic rhinitis, atopic dermatitis (AD) and atopic asthma. In this review, we aimed to discuss the biological characteristics of IL-21 and summarize the current progress on the role of IL-21 in the regulation of allergic inflammation.
\end{abstract}

\section{Contents}

1. Introduction

2. IL-21 and IL-21 receptor expression and signaling

3. IL-21 regulation of B-cell function

4. IL-21 in allergic inflammatory diseases

5. Conclusion

\section{Introduction}

Allergic disorders, such as allergic rhinitis, atopic dermatitis (AD) and atopic asthma, exhibit an inherited predisposition to sensitization by commonly encountered environmental allergens and to the development of high levels of immunoglobulin (Ig) E antibodies (1). It has been long recognized that Th2 lymphocytes and classical Th2 cell-derived cytokines, namely interleukin (IL)-4, IL-5 and IL-13, play an important role in orchestrating and amplifying allergic inflammation. Recently, several lines of evidence suggested that the tissue levels of IL-21 and the IL-21 receptor were elevated in patients with $\mathrm{AD}(2,3)$. In addition, tissue damage may be efficiently inhibited in murine models

Correspondence to: Dr Fang Gong, Department of Laboratory Medicine, The Third Hospital Affiliated to Nantong University, 585 North Xingyuan Road, Wuxi, Jiangsu 214041, P.R. China E-mail: gongfang2004@aliyun.com

Key words: interleukin-21, atopic dermatitis, allergic rhinitis, atopic asthma of AD and allergic rhinitis by dampening IL-21 levels (4), suggesting the involvement of IL-21 in the pathogenesis of these diseases. Moreover, an association of IL-21 polymorphisms with the susceptibility to atopic asthma was reported (5). In this review, we aimed to discuss the biological characteristics of IL-21 and summarize the current progress on the role of IL-21 in the regulation of allergic inflammation.

\section{IL-21 and IL-21 receptor expression and signaling}

IL-21 is a recently discovered member of the type I cytokine family, which is produced by activated CD $4^{+} \mathrm{T}$ cells, NKT cells, Th17 cells and follicular helper T cells $(6,7)$. The biological functions of IL-21 are mediated by a heterodimeric receptor, formed by a common $\gamma$-chain subunit, which is shared with the IL-2, IL-4, IL-7, IL-9 and IL-15 receptors, and its own unique receptor (designated IL-21R), a member of the class I cytokine receptor family (Fig. 1) (7,8). Although IL-21 production is restricted to lymphoid populations, IL-21R is highly expressed on a range of immune (i.e., naïve and activated T cells, B cells, NK cells, dendritic cells and macrophages), as well as non-immune cells (i.e., keratinocytes and endothelial cells) $(7,8)$, indicating a broad spectrum of functions. Of note, the expression of IL-21R on B cells was the highest, even on resting cells (1). Similar to other cytokines that signal through the common $\gamma$-chain, IL-21 functions by activating the Janus kinases JAK1 and JAK3, with JAK1 binding to IL-21R and JAK3 binding to the common $\gamma$-chain (6). IL-21R/ $\gamma_{c}$-driven signaling leads to JAK1 and JAK3 autophosphorylation and subsequent phosphorylation of signal transducer and activator of transcription (STAT) 3, STAT1, STAT5a and STAT5b (Fig. 1) (6,9). Among these STAT proteins, STAT3 appears to be the most important for IL-21 signaling, since the lack of expression of STAT3 leads to defective IL-21 signaling in $\mathrm{T}$ cells (9). Of note, the proliferation remained markedly decreased in STAT knockout (KO) mice, suggesting the involvement of other pathways in IL-21-mediated proliferation. Indeed, the phosphatidylinositol 3-kinase (PI3K)/Akt and mitogen-activated protein kinase (MAPK) pathways, together with the JAK/STAT pathway, cooperatively contribute to the full IL-21-mediated proliferative response (9).

\section{IL-21 regulation of $B$-cell function}

B cells contribute to the immunoreactivity of allergic diseases by giving rise to high titers of immunoglobulin (Ig) $\mathrm{E}$ 


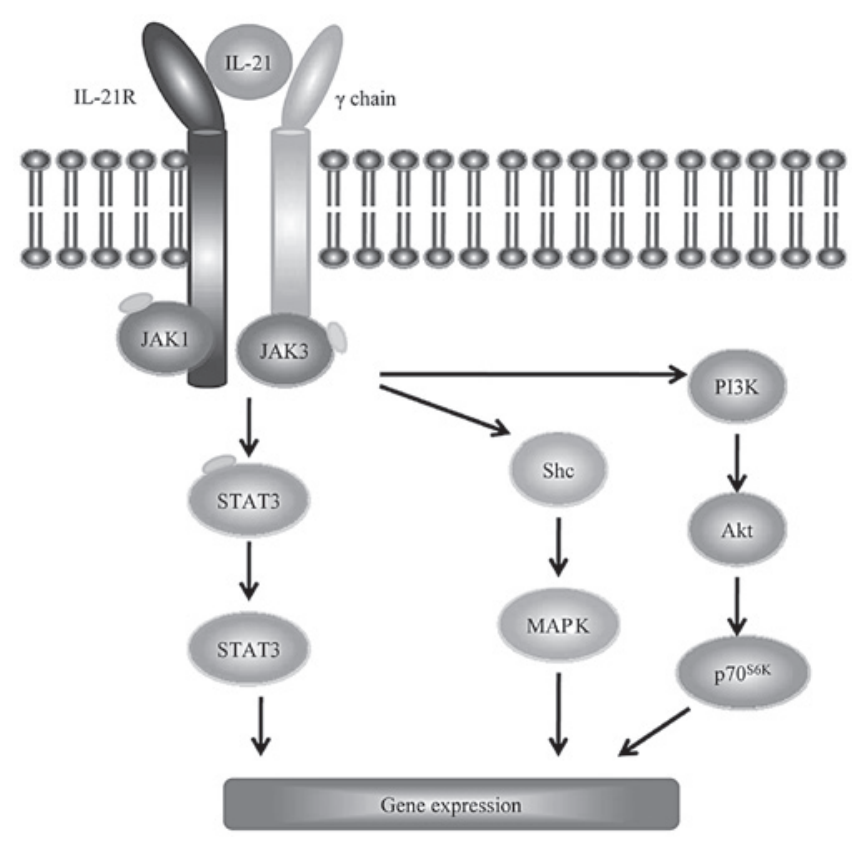

Figure 1. Schematic representation of the interleukin (IL)-21 receptor signaling pathway. IL-21R/ $/ \gamma_{c}$-driven signaling may activate three pathways, namely the JAK/STAT, PI3K/Akt and MAPK pathways. JAK, Janus-activated kinase; STAT, signal transducer and activator of transcription; PI3K, phosphatidylinositol 3-kinase; MAPK, mitogen-activated protein kinase.

antibodies. It was reported that IL-21 may not be essential for B-cell development, as IL-21R KO mice exhibited no defects in B-cell subsets within the bone marrow or in the periphery (10). However, IL-21 was shown to induce apoptosis, proliferation and class-switching in mature B-cell populations, depending on the co-stimulatory signals $(11,12)$. In the absence of a specific antigen or in the presence of a non-specific polyclonal signal, IL-21 induces apoptosis of naïve B cells, whereas IL-21 induces proliferation, isotype class-switching and differentiation to memory $\mathrm{B}$ cells or terminally differentiated plasma cells in the presence of a B-cell receptor signal and/or co-stimulatory interactions with $\mathrm{T}$ cells. In the absence of IL-21, the antibody isotype distribution is also disrupted. Naïve IL-21R KO mice exhibited diminished levels of IgG1, $\mathrm{IgG} 2 \mathrm{~b}$ and $\mathrm{IgG} 3$ and significantly higher levels of $\operatorname{IgE}$ upon immunization with T-cell-dependent antigens (10). This finding is consistent with results from in vivo and in vitro studies on wild-type mice: IL-21 administered to wild-type mice at the time of immunization may lead to reduced IgE responses and previous in vitro experiments demonstrated that IL-21 may reduce the levels of germline $\mathrm{C} \varepsilon$ transcripts, leading to reduced IgE-specific switching (13). The IL-21-mediated downregulation of IgE may result from the IL-21-induced expression of the pro-apoptotic Bcl-2-modifying factor in IgE-expressing $B$ cells (14), the induction of inhibitor of differentiation-2 in $\mathrm{B}$ cells or the reduction of germline $\mathrm{C} \varepsilon$ transcripts, leading to reduced IgE-specific switching (15).

\section{IL-21 in allergic inflammatory diseases}

IL-21 in allergic rhinitis. Allergic rhinitis represents the prototypical chronic rhinitis diseases. The typical clinical manifestations are nasal itching, sneezing, nasal running and nasal obstruction. Currently, allergic rhinitis is considered to be a disease mediated by IgE-mediated inflammation of the nasal mucosa, resulting in eosinophilic and Th2-cell infiltration of the nasal lining (16). Several cytokines produced by Th2 cells (e.g., IL-3, IL-4, IL-5 and IL-13) contribute to the induction and maintenance of the IgE production by plasma cells. Due to its ability to regulate IgE production, IL-21 is involved in the regulation of IgE-mediated allergic rhinitis responses. Indeed, in an ovalbumin-induced mouse model of allergic rhinitis, the intranasal administration of recombinant mouse IL-21 during the initial antigen challenge significantly reduced the allergic symptoms, with diminished antigen-specific serum IgE and reduced Th2 cytokine (IL-4, IL-5 and IL-13) levels in nasal tissues (4). Moreover, IL-21 acted on nasal fibroblasts to inhibit the production of eotaxin, leading to suppressed eosinophil migration into nasal tissues (4). To the best of our knowledge, there is only one available prospective study addressing this subject in humans. Huang et al (17) indicated there was no significant difference in the serum IL-21 levels between allergic rhinitis patients and healthy controls and that IL-21 was not associated with serum-specific IgE. However, due to the limited sample of only 24 patients and the fact that IL-21 was detected only at the protein level, further studies, involving a larger sample size, are required to investigate the precise role of IL-21 in allergic rhinitis at the mRNA level.

IL-21 in $A D . \mathrm{AD}$ is a pruritic allergic inflammatory skin disease, frequently associated with high plasma levels of $\operatorname{IgE}(18)$. AD affects $15-30 \%$ of children and $2-10 \%$ of the adult population worldwide (19). Although AD has been the subject of numerous investigations, the pathophysiology of this disease has not been fully elucidated (19).

Recently, a study by Jin et al (3) identified IL-21 as a critical regulator of the processes that lead to sensitization and allergic inflammation of the skin. By using a mouse model of allergic 
skin inflammation, Jin et al (3) demonstrated that the gene expression levels of IL-21 and IL-21R were significantly upregulated in mouse skin subjected to tape stripping, a surrogate for scratching. Moreover, IL-21R deficient and wild-type mice treated with soluble IL-21R-IgG2aFc fusion protein developed an impaired systemic response to epicutaneous sensitization and disrupted trafficking of skin dendritic cells (3). Further insight into this defect was provided by the observation of impaired migration of skin dendritic cells towards draining lymph nodes following antigen capture in IL-21R-deficient mice. In addition, the expression of CCR7/TARC by skin dendritic cells and MMP2 activation in the epidermis following mechanical injury were hindered (3). These data offer insight into a potential mechanism underlying the association of IL-21 signaling with the pathogenesis of allergic dermatitis and contributing to allergic skin inflammation.

As regards humans, elevated protein expression levels of IL-21 and IL-21R were detected in acute skin lesions of patients suffering from AD (3). However, a previous study by Lin et al (20) revealed that the serum levels of IL-21 in AD patients were significantly lower compared to those in healthy controls. In addition, it was reported that serum IL-21 levels were inversely correlated with the severity of AD (20). The severity of the skin manifestations was inversely correlated with the serum IL-21 titers. The differences in IL-21 expression between the skin lesions and the serum may be attributed to the uneven distribution, disease duration, or treatment regimen applied.

IL-21 in atopic asthma. Atopic asthma (also referred to as allergic asthma), is a chronic airway inflammatory disease primarily characterized by an abnormality in the $\operatorname{IgE}$ pathway, which is associated with the inception of asthma and its acute deterioration (21). Evidence supporting a genetic basis for asthma was obtained from recent research including case-control and family studies (5). It was reported that the exon-3 polymorphism C5250T of the IL-21 gene is significantly associated with atopic asthma and serum total $\mathrm{IgE}$. Furthermore, the C5250T polymorphism was shown to affect the concentration of serum IL-21 in atopic asthmatics (5). Of note, IL-21R-deficient mice exhibited unexpectedly reduced airway hyperresponsiveness, although the serum IgE levels were increased (22), although recent results obtained from animal models suggested that IL-21 may diminish the severity of allergy (4,5). A possible explanation for this discrepancy is that IL-21 may play different roles, depending on the immune setting and the combination with different cytokines. Although further functional studies are required, it may be hypothesized that IL-21 is involved in the pathogenesis of atopic asthma.

\section{Conclusion}

The pleiotropic inflammatory functions of IL-21 and the documented pathogenic effects of this cytokine in various tissues suggest that the interruption of the IL-21 signaling pathway merits extensive investigation as a therapeutic option for the treatment of inflammatory and allergic diseases. However, mouse models do not reflect the full spectrum of the complexity of human disease. Therefore, further research is required to achieve therapeutic efficacy in humans.

\section{References}

1. Galli SJ, Tsai M and Piliponsky AM: The development of allergic inflammation. Nature 454: 445-454, 2008.

2. Caruso R, Botti E, Sarra M, et al: Involvement of interleukin-21 in the epidermal hyperplasia of psoriasis. Nat Med 15: 1013-1015, 2009.

3. Jin H, Oyoshi MK, Le Y, et al: IL-21R is essential for epicutaneous sensitization and allergic skin inflammation in humans and mice. J Clin Invest 119: 47-60, 2009.

4. Hiromura Y, Kishida T, Nakano H, Hama T, Imanishi J, Hisa Y and Mazda O: IL-21 administration into the nostril alleviates murine allergic rhinitis. J Immunol 179: 7157-7165, 2007.

5. Chatterjee R, Batra J and Ghosh B: A common exonic variant of interleukin 21 confers susceptibility to atopic asthma. Int Arch Allergy Immunol 148: 137-146, 2009.

6. Crotty S: Follicular helper CD4 T cells (TFH). Annu Rev Immunol 29: 621-663, 2011.

7. Spolski R and Leonard WJ: Interleukin-21: basic biology and implications for cancer and autoimmunity. Annu Rev Immunol 26: 57-79, 2008

8. Sarra M, Cupi ML, Pallone F and Monteleone G: Interleukin-21 in immune and allergic diseases. Inflamm Allergy Drug Targets 11: 313-319, 2012.

9. Zeng R, Spolski R, Casas E, Zhu W, Levy DE and Leonard WJ: The molecular basis of IL-21-mediated proliferation. Blood 109: 4135-4142, 2007

10. Ozaki K, Spolski R, Feng CG, et al: A critical role for IL-21 in regulating immunoglobulin production. Science 298: 1630-1634, 2002.

11. Mehta DS, Wurster AL, Whitters MJ, Young DA, Collins M and Grusby MJ: IL-21 induces the apoptosis of resting and activated primary B cells. J Immunol 170: 4111-4118, 2003.

12. Jin H, Carrio R, Yu A and Malek TR: Distinct activation signals determine whether IL-21 induces B cell costimulation, growth arrest, or Bim-dependent apoptosis. J Immunol 173: 657-665, 2004.

13. Suto A, Nakajima H, Hirose K, et al: Interleukin 21 prevents antigen-induced IgE production by inhibiting germ line $\mathrm{C} \varepsilon$ transcription of IL-4-stimulated B cells. Blood 100: 4565-4573, 2002.

14. Harada M, Magara-Koyanagi K, Watarai H, et al: IL-21-induced $\mathrm{B} \varepsilon$ cell apoptosis mediated by natural killer $\mathrm{T}$ cells suppresses IgE responses. J Exp Med 203: 2929-2937, 2006.

15. Kishida T, Hiromura Y, Shin-Ya M, et al: IL-21 induces inhibitor of differentiation 2 and leads to complete abrogation of anaphylaxis in mice. J Immunol 179: 8554-8561, 2007.

16. Small P and Kim H: Allergic rhinitis. Allergy Asthma Clin Immunol 7 (Suppl 1): S3, 2011.

17. Huang X, Yang Q, Chen Y, Li P, Zhang G and Li Y: Expressions of IL-17, IL-21 and IL-23 in the serum of allergic rhinitis patients. J Med Biochem 30: 323-327, 2011

18. Hayashida S, Uchi H, Moroi Y and Furue M: Decrease in circulating Th17 cells correlates with increased levels of CCL17, IgE and eosinophils in atopic dermatitis. J Dermatol Sci 61: 180-186, 2011.

19. Bieber T: Atopic dermatitis. N Engl J Med 358: 1483-1494, 2008.

20. Lin SC, Chuang YH, Yang YH and Chiang BL: Decrease in interleukin-21 in children suffering with severe atopic dermatitis. Pediatr Allergy Immunol 22: 869-875, 2011.

21. Wenzel SE: Asthma phenotypes: the evolution from clinical to molecular approaches. Nat Med 18: 716-725, 2012.

22. Fröhlich A, Marsland BJ, Sonderegger I, Kurrer M, Hodge MR, Harris NL and Kopf M: IL-21 receptor signaling is integral to the development of Th2 effector responses in vivo. Blood 109: 2023-2031, 2007. 